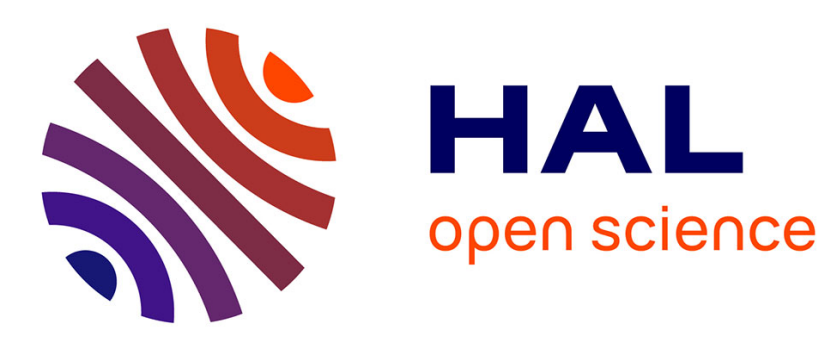

\title{
MTTF Estimation Using Importance Sampling on Markov Models
}

Héctor Cancela, Gerardo Rubino, Bruno Tuffin

\section{To cite this version:}

Héctor Cancela, Gerardo Rubino, Bruno Tuffin. MTTF Estimation Using Importance Sampling on Markov Models. [Research Report] RR-3672, INRIA. 1999. inria-00073000

\section{HAL Id: inria-00073000 \\ https://hal.inria.fr/inria-00073000}

Submitted on 24 May 2006

HAL is a multi-disciplinary open access archive for the deposit and dissemination of scientific research documents, whether they are published or not. The documents may come from teaching and research institutions in France or abroad, or from public or private research centers.
L'archive ouverte pluridisciplinaire HAL, est destinée au dépôt et à la diffusion de documents scientifiques de niveau recherche, publiés ou non, émanant des établissements d'enseignement et de recherche français ou étrangers, des laboratoires publics ou privés. 
INSTITUT NATIONAL DE RECHERCHE EN INFORMATIQUE ET EN AUTOMATIQUE

\section{MTTF estimation using importance sampling on Markov models}

Héctor CANCELA, Gerardo RUBINO and Bruno TUFFIN

\section{$\mathbf{N}^{\circ} 3672$}

April 29, 1999

THÈME 1 



\title{
MTTF estimation using importance sampling on Markov models
}

\author{
Héctor CANCELA*, Gerardo RUBINO ${ }^{\dagger}$ and Bruno TUFFIN ${ }^{\ddagger}$ \\ Thème 1 - Réseaux et systèmes \\ Projet Armor
}

Rapport de recherche n3672 - April 29, 1999 - 29 pages

\begin{abstract}
Very complex systems occur nowadays quite frequently in many technological areas and they are often required to comply with high dependability standards. To study their availability and reliability characteristics, Markovian models are commonly used. Due to the size and complexity of the systems, and due to the rarity of system failures, both analytical solutions and "crude" simulation can be inefficient or even non-relevant. A number of variance reduction Monte Carlo techniques have been proposed to overcome this difficulty; importance sampling methods are among the most efficient. The objective of this paper is to survey existing importance sampling schemes, to propose some improvements and to discuss on their different properties.
\end{abstract}

Key-words: Rare event simulation, variance reduction, Markovian Models, dependability measures.

(Résumé : tsvp)

* UDELAR, Montevideo, Uruguay, cancela@fing.edu.uy

${ }^{\dagger}$ ENSTB, Cesson-Sevigné, France \& IRISA, Rennes, France, rubino@irisa.fr

‡ IRISA, Rennes, France, btuffin@irisa.fr 


\section{Estimation de la MTTF utilisant l'échantillonnage préférentiel sur des modèles Markoviens}

Résumé : Des systèmes très complexes interviennent de nos jours fréquemment dans beaucoup de domaines technologiques et doivent souvent offrir une importante sûreté de fonctionnement. Pour étudier leurs caractéristiques de disponibilité et de fiabilité, les modèles Markoviens sont communément utilisés. En raison de la taille et de la complexité de ces systèmes, et en raison de la rareté des défaillances, les solutions analytiques et la simulation "standard" sont toutes deux inefficaces, et même parfois non applicables. Un certain nombre de techniques de Monte Carlo réduisant la variance ont été proposées pour surmonter cette difficulté; les méthodes d'échantillonnage préférentiel sont parmi les plus efficaces. L'objectif de cet article est de passer en revue les procédés d'échantillonnage préférentiel existants, de proposer des améliorations et de discuter des différentes propriétés de ces méthodes.

Mots-clé : Simulation d'événements rares, réduction de la variance, modèles Markoviens, mesures de sûreté de fonctionnement 


\section{Introduction}

Let us consider a multi-component repairable system. The user has defined what an operational state is, and the fact that the system is repairable means that it can come back at such a state after the occurrence of a failure, due to some repairing facility included in. We are interested in evaluating some specific dependability metrics from a model of the system. If $X_{t}$ denotes the state of the model at time $t$, where $X_{t} \in S$, the specifications induce a partition of the state space $S$ into two (disjoint) sets: $U$, the set of states where the system is up (delivering service as specified), and $D$, composed by those states where the system is down (the delivered service does not fit anymore the specifications).

The most important dependability metrics are: (i) the asymptotic availability, defined by $\operatorname{Pr}\left(X_{\infty} \in U\right.$ ) (assuming for instance that the model is irreducible and ergodic); (ii) the MTTF (Mean Time To Failure), defined as $\mathrm{E}\left(\tau_{D}\right)$ where $\tau_{D}$ is the hitting time of the set $D$ (assuming that $X_{0} \in U$ ), that is, $\tau_{D}=\inf \left\{t \mid X_{t} \in D\right\}$; (iii) the reliability at time $t$, equal to $\operatorname{Pr}\left(\tau_{D}>t\right.$ ), also assuming that $X_{0} \in U$; (iv) the point availability at time $t$, defined by $\operatorname{Pr}\left(X_{t} \in U\right) ;(\mathrm{v})$ the distribution (or the moments) of the random variable interval availability in $[0, t]$, defined by $\frac{1}{t} \int_{0}^{t}\left[X_{s} \in U\right] d s$ where $[\mathcal{P}]$ is the indicator function of the predicate $\mathcal{P}$.

A frequent situation is that the model (the stochastic process $X$ ) is quite complex and large (that is, $|S| \gg 1$ ) and that the failed states are rare, that is, $\tau_{D} \gg \tau_{0}$ with high probability, where $\tau_{0}$ is the return time to the initial state 0 , presumed to be the (only) state where all the components are up (that is, $\left.\tau_{0}=\inf \left\{t>0 \mid X_{t}=0, X_{t^{-}} \neq 0\right\}\right)$. The size of the model may make difficult or impossible its exact numerical evaluation and the rarity of the interesting events can do the same with a naive Monte Carlo estimation [6]. In the first case, an alternative approach deals with the computation of bounds of the measures of interest. In this line, see [11] for an efficient scheme devoted to the analysis of the asymptotic availability, extended in [10] to deal with more general models (and also to the analysis of asymptotic performance measures). In the Monte Carlo area, different importance sampling schemes have been proven to be appropriate, in order to design efficient estimation algorithms. This paper focuses on a basic and widely used dependability measure, the

RR n 3672 
MTTF. We analyze some known importance sampling schemes designed to estimate it, we exhibit some improving techniques and we discuss on general properties of this family of methods.

This paper is organized as follows. We give the model specifications in Section 2 and we describe general simulation techniques in Section 3. As we study highly dependable systems, we introduce a rarity parameter in Section 4 and we present the importance sampling schemes in Section 5. Section 6 deals with important properties of the estimators: bounded relative error and bounded normal approximation. Comparisons of the algorithms are then given: in Section 7 asymptotically as the rarity parameter goes to 0, and numerically in Section 8. Moreover we show in Section 9 that numerical results can lead to wrong estimations in some cases and we conclude in Section 10.

\section{The model}

The system is represented (modeled) by a finite continuous time homogeneous and irreducible Markov chain $X=\left\{X_{t}, t \geq 0\right\}$. We denote by $S$ the state space of $X$, and we suppose that $1 \ll|S|(<\infty)$.

Let us denote by $Q(x, y)$ the transition rate from state $x$ to state $y$ and by $Y$ the discrete time homogeneous and irreducible Markov chain canonically embedded in $X$ at its jump times. The transition probability $P(x, y)$ that $Y$ visits state $y$ after state $x$ verifies

$$
P(x, y)=\frac{Q(x, y)}{\sum_{z: z \neq x} Q(x, z)} .
$$

Let us precise here the main characteristics of the model. We assume that the components are (i) either operational (or up), or (ii) unoperational (or down, that is, failed). The same happens with the whole system. As said before, $S=U \cup D$ where $U$ is the set of up states and $D$ is the set of down states, $U \cap D=\emptyset$. The components have also a class or type belonging to the set $\mathcal{K}=\{1,2, \cdots, K\}$ of classes. An operational class $k$ component has failure rate $\lambda_{k}(x)$ when the model is in state $x$.

In the sequel, we will basically follow the notation used in [14] and [12] and the assumptions made there. The whole set of transitions is partitioned into 
two (disjoint) sets $\mathcal{F}$, the set of failures and $\mathcal{R}$, the set of repairs. To facilitate the reading, we denote $Q(x, y)=\lambda(x, y)$ when $(x, y) \in \mathcal{F}$ and $Q(x, y)=\mu(x, y)$ when $(x, y) \in \mathcal{R}$. We also denote by $F_{x}$ the set of states that can be reached from $x$ after a failure, and by $R_{x}$ the set of states that can be reached from $x$ after a repair, that is,

$$
F_{x}=\{y \mid(x, y) \in \mathcal{F}\}, \quad R_{x}=\{y \mid(x, y) \in \mathcal{R}\} .
$$

Recall that we assume that the initial state is fixed and denoted by 0 . Since all the components are up in that state, we assume $0 \in U$. We also have $R_{0}=\emptyset$ (that is, no repairs from 0 since everything is assumed to work when the system's state is 0$)$.

Let us denote by $\nu_{k}(x)$ the number of operational components of class $k$ when the model state is $x$. The intuitive idea of failure and repair translate into the following formal relationships: for all $x \in S$,

$$
\begin{aligned}
& (x, y) \in \mathcal{F} \Longleftrightarrow \forall k, \nu_{k}(x) \geq \nu_{k}(y) \text { and } \exists k \text { s.t. } \nu_{k}(x)>\nu_{k}(y), \\
& (x, y) \in \mathcal{R} \Longleftrightarrow \forall k, \nu_{k}(x) \leq \nu_{k}(y) \text { and } \exists k \text { s.t. } \nu_{k}(x)<\nu_{k}(y) .
\end{aligned}
$$

To finish the description of the model, let us specify how the transitions occur. After the failure of some operational class $k$ component when the system state is $x$, the system jumps to state $y$ with probability $p(y ; x, k)$. This allows to take into account the case of failure propagation, that is, the situation where the failure of some component induces, with some probability, that a subset of components is shut down (for instance, the failure of the power supply can make some other components unoperational). The probabilities $p(y ; x, k)$ are assumed to be defined for all $y, x, k$; in general, in most of the cases $p(y ; x, k)=0$.

Observe that

$$
\forall(x, y) \in \mathcal{F}, \quad \lambda(x, y)=\sum_{k=1}^{K} \nu_{k}(x) \lambda_{k}(x) p(y ; x, k) .
$$

Concerning the repairs, the only needed assumption is that from every state different from the initial one, there is at least one repair transition, that is,

$$
\forall x \neq 0, R_{x} \neq \emptyset \text {. }
$$

This excludes the case of delayed repairs, corresponding to systems where the repair facilities are activated only when there are "enough" failed units.

RR n3672 


\section{Regenerative Monte Carlo scheme}

The regenerative approach to evaluate the MTTF consists of using the following expression:

$$
M T T F=\frac{\mathrm{E}\left(\min \left(\tau_{D}, \tau_{0}\right)\right)}{\gamma}
$$

where $\gamma=\operatorname{Pr}\left(\tau_{D}<\tau_{0}\right)$ [5]. To estimate $\mathrm{E}\left(\min \left(\tau_{D}, \tau_{0}\right)\right)$ and $\gamma$, we generate independent cycles $C_{1}, C_{2}, \ldots$, that is, sequences of adjacent states starting and ending with state 0 , and not containing it in any other position, and we estimate the corresponding expectations.

Observe first that the numerator and the denominator in the r.h.s. of (1) can be computed directly from the embedded discrete time chain $Y$, that is, working in discrete time. To formalize this, let us denote by $\mathcal{C}$ the set of all the cycles and by $\mathcal{D}$ the set of the cycles passing through $D$. The probability of a cycle $c \in \mathcal{C}$ is

$$
q(c)=\prod_{(x, y) \in c} P(x, y) .
$$

An estimator of $M T T F$ is then

$$
\widehat{M T T} F=\frac{\sum_{i=1}^{I} G\left(C_{i}\right)}{\sum_{i=1}^{I} H\left(C_{i}\right)}
$$

where for any cycle $c$, we define $G(c)$ as the sum of the expectations of the sojourn times in all its states until reaching $D$ or being back to 0 , and $H(c)$ is equal to 1 if $c \in \mathcal{D}$, and to 0 otherwise. Observe that, to estimate the denominator in the expression of the $M T T F$, when a cycle reaches $D$, the path construction is stopped since we already know that $\tau_{D}<\tau_{0}$.

Using the Central Limit Theorem, we have [5]

$$
\frac{\sqrt{I}(\widehat{M T T} F-M T T F)}{\sigma / \bar{H}_{I}} \rightarrow N(0,1)
$$

with $\bar{H}_{I}=\frac{1}{I} \sum_{i=1}^{I} H\left(C_{i}\right)$, and

$$
\sigma^{2}=\sigma_{q}^{2}(G)-2 M T T F \operatorname{Cov}_{q}(G, H)+M T T F^{2} \sigma_{q}^{2}(H),
$$


where $\sigma_{q}^{2}(F)$ denotes the variance of $F$ under the probability measure $q$. A confidence interval can thus be obtained.

The estimation of the numerator in (1) presents no problem even in the rare event context since in that case $\mathrm{E}\left(\min \left(\tau_{D}, \tau_{0}\right)\right) \approx \mathrm{E}\left(\tau_{0}\right)$. The estimation of $\gamma$, however, is difficult or even impossible using the standard Monte Carlo scheme in the rare event case. Indeed, the expectation of the first time that event " $\tau_{D}<\tau_{0}$ " occurs is about $1 / \gamma$, then large for highly reliable systems. For its estimation, we can follow an importance sampling approach. The idea is to change the underlying measure such that all the cycles in the interesting set $\mathcal{D}$ receive a higher weight. This is not possible in general, and what we in fact do is to change the transition probabilities $P()$ 's into $P^{\prime}()$ 's with an appropriate choice such that we expect that the weight $q^{\prime}()$ of most of the interesting cycles will increase.

The following method, called MS-DIS for Measure Specific Dynamic Importance Sampling and introduced in [5], uses independent simulations for the numerator and denominator of (1). On the total $I$ sample paths, $\xi I$ are reserved for the estimation of $E\left(\min \left(\tau_{D}, \tau_{0}\right)\right)$ and $(1-\xi) I$ for the estimation of $\gamma$. As the estimation of $E\left(\min \left(\tau_{D}, \tau_{0}\right)\right)$ is simple, we use the crude estimator $\bar{G}_{\xi I}=(\xi I)^{-1} \sum_{i=1}^{\xi I} G\left(C_{i, q}\right)$ where $C_{i, q}$, is the $i$ th path sampled under probability measure $q$. The importance sampling technique is applied to the estimation of $\gamma$. A new estimator of the $M T T F$ is then [5]

$$
\widehat{M T T F}=\frac{\bar{G}_{\xi I}}{\bar{H}_{(1-\xi) I}}
$$

with

$$
\bar{H}_{(1-\xi) I}=((1-\xi) I)^{-1} \sum_{i=1}^{(1-\xi) I} H\left(C_{i}\right) \frac{q\left(C_{i, q^{\prime}}\right)}{q^{\prime}\left(C_{i, q^{\prime}}\right)}
$$

using independent paths $C_{i, q^{\prime}}, 1 \leq i \leq(1-\xi) I$, sampled under the new probability measure $q^{\prime}$, and independent of the $C_{i, q}, 1 \leq i \leq \xi I$. We have then $[5]$

with

$$
\frac{\sqrt{I}(\widehat{M T T} F-M T T F)}{\sigma / \bar{H}_{(1-\xi) I}} \rightarrow N(0,1)
$$

$$
\sigma^{2}=\frac{\sigma_{q}^{2}(G)}{\xi}+(M T T F)^{2} \frac{\sigma_{q^{\prime}}^{2}\left(H q / q^{\prime}\right)}{1-\xi} .
$$

RR n 3672 
A dynamic choice of $\xi$ can also be made to reduce $\sigma^{2}$.

In Section 5, we review the main schemes proposed for the estimation of $\gamma$, and we propose some new ways of performing the estimations, which will be shown to behave better in appropriate situations. Next section first discuss the formalization of the rare event situation, in order to be able to develop the analysis of those techniques.

\section{The rarity parameter}

We must formalize the fact that failures are rare or slow, and that repairs are fast. Following [14], we introduce a rarity parameter $\varepsilon$. We assume that the failures rates of class $k$ components have the following form:

$$
\lambda_{k}(x)=a_{k}(x) \varepsilon^{i_{k}(x)}
$$

where either the real $a_{k}(x)$ is strictly positive and the integer $i_{k}(x)$ is greater than or equal to 1 , or $a_{k}(x)=i_{k}(x)=0$. To simplify things, we naturally set $a_{k}(x)=0$ if $\nu_{k}(x)=0$. No particular assumption is necessary about the $p(y ; x, k)$ 's, so, we write

$$
p(y ; x, k)=b_{k}(x, y) \varepsilon^{j_{k}(x, y)}
$$

with real $b_{k}(x, y) \geq 0$, integer $j_{k}(x, y) \geq 0$, and $j_{k}(x, y)=0$ when $b_{k}(x, y)=0$. Concerning the repair rates, we simply state

$$
\mu(x, y)=\Theta(1)
$$

where $f(\varepsilon)=\Theta\left(\varepsilon^{d}\right)$ means that there exists two constants $k_{1}, k_{2}>0$ such that $k_{1} \varepsilon^{d} \leq|f(\varepsilon)| \leq k_{2} \varepsilon^{d}$ (recall that for every state $x \neq 0$, there exists at least one state $y$ s.t. $\mu(x, y)>0)$. We can thus observe that the rarity of the interesting event " $\tau_{D}<\tau_{0}$ " increases when $\varepsilon$ decreases.

The form of the failure rates of the components has the following consequence on the failure transitions in $X$ : for all $(x, y) \in \mathcal{F}$,

$$
\lambda(x, y)=\Theta\left(\varepsilon^{m(x, y)}\right)
$$


where

$$
m(x, y)=\min _{k: a_{k}(x) b_{k}(x, y)>0}\left\{i_{k}(x)+j_{k}(x, y)\right\}
$$

(observe that if $F_{x} \neq \emptyset$, then for all $y \in F_{x}$ we necessarily have $m(x, y) \geq 1$ ).

Let us look now at the transition probabilities of $Y$. For any $x \neq 0$, since we assume that $R_{x} \neq \emptyset$, we have

$$
(x, y) \in \mathcal{F} \Longrightarrow P(x, y)=\Theta\left(\varepsilon^{m(x, y)}\right), m(x, y) \geq 1,
$$

and

$$
(x, y) \in \mathcal{R} \Longrightarrow P(x, y)=\Theta(1) \text {. }
$$

For the initial state, we have that for all $y \in F_{0}$,

$$
P(0, y)=\Theta\left(\varepsilon^{m(0, y)-\min _{z \in F_{0}} m(0, z)}\right) .
$$

Observe here that if $\operatorname{argmin}_{z \in F_{0}} m(0, z)=w \in D$, then we have $P(0, w)=\Theta(1)$ and there is no rare event problem. This happens in particular if $F_{0} \cap U=\emptyset$. So, the interesting case for us (the rare event situation) is the case of $P(0, w)=o(1)$ for all $w \in F_{0} \cap D$. In other words, the case of interest is when (i) $F_{0} \cap U \neq \emptyset$ and (ii) $\exists y \in F_{0} \cap U$ s.t. $\forall \omega \in F_{0} \cap D, m(0, y)<m(0, \omega)$.

A simple consequence of the previous assumptions is that for any cycle $c$, its probability $q(c)$ is $q(c)=\Theta\left(\varepsilon^{h}\right)$ where the integer $h$ is $h \geq 1$. If we define

$$
\mathcal{C}_{h}=\left\{c \in \mathcal{C} \mid q(c)=\Theta\left(\varepsilon^{h}\right)\right\}
$$

then we have [14]

$$
\gamma=\sum_{c \in \mathcal{D}} q(c)=\Theta\left(\varepsilon^{r}\right)
$$

where $r=\operatorname{argmin}\left\{h \mid \mathcal{C}_{h} \neq \emptyset\right\} \geq 1$. We see formally now that $\gamma$ decreases as $\varepsilon \rightarrow 0$.

\section{$5 \quad$ Importance sampling schemes}

To simplify the description of the different schemes, let us introduce the following notation. For all state $x$, we denote by $f_{x}(y)$ the transition probability

RR n 3672 
$P(x, y)$, for each $y \in F_{x}$. In the same way, for all state $x$, let us denote $r_{x}(y)=P(x, y)$ for each $y \in R_{x}$. Using an importance sampling scheme means that instead of $P$ we use a different matrix $P^{\prime}$, leading to new $f_{x}^{\prime}()$ 's and $r_{x}^{\prime}()^{\prime}$ 's. The transition probabilities associated with the states of $D$ are not concerned in the estimation of $\gamma$ since when a cycle reaches $D$, it is "stopped" as we explained in Section 3.

\subsection{Failure biasing (FB) [8] [4]}

This is the most straightforward method: to increase the probability of regenerative cycles including system failures, we increase the probability of the failure transitions. We must choose a parameter $\alpha \in(0,1)$, which is equal to $f_{x}^{\prime}\left(F_{x}\right)$ for all $x \neq 0$ (typically, $0.5 \leq \alpha \leq 0.9$ ). The transition probabilities are then changed as follows.

- $\forall x \in U, x \neq 0, \forall y \in F_{x}: \quad f_{x}^{\prime}(y)=\alpha \frac{f_{x}(y)}{f_{x}\left(F_{x}\right)}$

- $\forall x \in U, x \neq 0, \forall y \in R_{x}: \quad r_{x}^{\prime}(y)=(1-\alpha) \frac{r_{x}(y)}{r_{x}\left(R_{x}\right)}$.

The $f_{0}()$ 's are not modified (since we already have $f_{0}\left(F_{0}\right)=\Theta(1)$ ). Observe that the total probability of failure from $x$ is now equal to $\alpha$ (that is, for any $\left.x \in U-\{0\}, f_{x}^{\prime}\left(F_{x}\right)=\alpha\right)$.

\subsection{Selective failure biasing (SFB)[5]}

The idea here is to separate the failure transitions from $x(x \in U)$ into two disjoint sets: those consisting of the first failure of a component of some class $k$ (and called initial failures), and the remaining ones (called non-initial failures). Following this, the set of states $F_{x}$ is partitioned into two (disjoint) sets $I F_{x}$ and $N I F_{x}$, where

$$
\begin{gathered}
I F_{x}=\{y \mid(x, y) \text { is an initial failure }\}, \\
N I F_{x}=\{y \mid(x, y) \text { is a non-initial failure }\} .
\end{gathered}
$$


The idea is then to increase the probability of a non-initial failure, that is, to make the failure of some class $k$ components more probable than in the original model, if there is at least one component of that class that has already failed.

To implement this, we must choose two parameters $\alpha, \beta \in(0,1)$ (typically, $0.5 \leq \alpha, \beta \leq 0.9)$ and change the transition probabilities in the following way:

- $\forall x \in U, x \neq 0, \forall y \in I F_{x}, \quad f_{x}^{\prime}(y)=\alpha(1-\beta) \frac{f_{x}(y)}{f_{x}\left(I F_{x}\right)}$ and $\forall y \in N I F_{x}, \quad f_{x}^{\prime}(y)=\alpha \beta \frac{f_{x}(y)}{f_{x}\left(N I F_{x}\right)}$

for $x=0$, we use the same formulae with $\alpha=1$; in the same way, if $I F_{x}=\emptyset$, we use $\beta=1$ and if $N I F_{x}=\emptyset$, we set $\beta=0$.

- $\forall x \in U, x \neq 0, \forall y \in R_{x}, \quad r_{x}^{\prime}(y)=(1-\alpha) \frac{r_{x}(y)}{r_{x}\left(R_{x}\right)}$.

In this scheme, as in the FB method, the total failure probability is $f_{x}^{\prime}\left(F_{x}\right)=$ $\alpha$, but now, we have a further refinement, leading to $f_{x}^{\prime}\left(N I F_{x}\right)=\alpha \beta$ and $f_{x}^{\prime}\left(I F_{x}\right)=\alpha(1-\beta)$.

\subsection{Selective failure biasing for "series-like" systems (SFBS)}

The implicit assumption in SFB is that the criteria used to define an operational state (that is, the type of considered system) is close to the situation where the system is up if and only if, for each component class $k$, the number of operational components is greater or equal some threshold $l_{k}$, and if neither the initial number of components $N_{k}$ nor the level $l_{k}$ are "very dependent" on $k$. Now, assume that this last part of the assumptions does not hold, that is, assume that from the dependability point of view, the system is a series of $l_{k}$-out-of- $N_{k}$ modules, but that the $N_{k}$ 's and the $l_{k}$ 's are strongly dependent on $k$. A reasonable way to improve $\mathrm{SFB}$ is to make more probable the failures of the class $k$ components for which $\nu_{k}(x)$ is closer to the threshold $l_{k}$.

Consider a state $x \in U$ and define a class $k$ critical in $x$ if $\nu_{k}(x)-l_{k}=$ $\min _{k^{\prime}=1, \cdots, K}\left(\nu_{k^{\prime}}(x)-l_{k^{\prime}}\right)$; otherwise, the class is non-critical. Now, for a state $y \in F_{x}$, the transition $(x, y)$ is critical if there is some critical class $k$ in $x$

RR n 3672 
such that $\nu_{k}(y)<\nu_{k}(x)$. We denote by $F_{x, c}$ the subset of $F_{x}$ composed of the critical failures, that is,

$$
F_{x, c}=\left\{y \in F_{x} \mid(x, y) \text { is critical }\right\} .
$$

We also define $F_{x, n c}$, the set of non-critical failures, by $F_{x, n c}=F_{x}-F_{x, c}$. Then, a specialized SFB method, which we call SFBS, can be defined by the following modification of the $f_{x}($ )'s (we omit the frontiers' case which is handled as for SFB):

- $\forall x \in U, \forall y \in F_{x, n c}, f_{x}^{\prime}(y)=\alpha(1-\beta) \frac{f_{x}(y)}{f_{x}\left(F_{x, n c}\right)}$,

$$
\begin{gathered}
\text { and } \forall y \in F_{x, c}, \quad f_{x}^{\prime}(y)=\alpha \beta \frac{f_{x}(y)}{f_{x}\left(F_{x, c}\right)} . \\
\text { - } \forall y \in R_{x}, \quad r_{x}^{\prime}(y)=(1-\alpha) \frac{r_{x}(y)}{r_{x}\left(R_{x}\right)} .
\end{gathered}
$$

See Section 7 for the numerical behavior of this method and the the gain that can be obtained when using it instead of SFB.

\subsection{Selective failure biasing for "parallel-like" systems (SFBP)}

This is the dual of SFBS. Think of a system working as sets of $l_{k}$-out-of- $N_{k}$ modules in parallel, $1 \leq k \leq K$. Consider a state $x \in U$ and define a class $k$ critical in $x$ if $\nu_{k}(x) \geq l_{k}$; otherwise, the class is non-critical. Now, for a state $y \in F_{x}$, the transition $(x, y)$ is critical if there is some critical class $k$ in $x$ such that $\nu_{k}(y)<\nu_{k}(x)$. As before, the set of states $y \in F_{x}$ such that $(x, y)$ is critical, is denoted by $F_{x, c}$, and $F_{x, n c}=F_{x}-F_{x, c}$.

A first idea is to follow the analogous scheme as for the SFBS case: using in the same way two parameters $\alpha$ and $\beta$, the principle would be to accelerate the critical transitions first, then the non-critical ones, by means of the respective weights $\alpha \beta$ and $\alpha(1-\beta)$. This leads to the following rules:

- $\forall x \in U, \forall y \in F_{x, n c}, f_{x}^{\prime}(y)=\alpha(1-\beta) \frac{f_{x}(y)}{f_{x}\left(F_{x, n c}\right)}$,

$$
\text { and } \forall y \in F_{x, c}, \quad f_{x}^{\prime}(y)=\alpha \beta \frac{f_{x}(y)}{f_{x}\left(F_{x, c}\right)} \text {. }
$$


- $\forall y \in R_{x}, \quad r_{x}^{\prime}(y)=(1-\alpha) \frac{r_{x}(y)}{r_{x}\left(R_{x}\right)}$

As we will see in Section 7, there is no need for the $\beta$ parameter, and the method we call SFBP is then defined by the following rules:

- $\forall x \in U, \forall y \in F_{x, c}, \quad f_{x}^{\prime}(y)=\alpha \frac{f_{x}(y)}{f_{x}\left(F_{x, c}\right)}$,

and $\forall y \in F_{x, n c}, \quad f_{x}^{\prime}(y)=(1-\alpha) \frac{f_{x}(y)}{r_{x}\left(F_{x}\right)+f_{x}\left(F_{x, n c}\right)}$.

- $\forall y \in R_{x}, \quad r_{x}^{\prime}(y)=(1-\alpha) \frac{r_{x}(y)}{r_{x}\left(F_{x}\right)+f_{x}\left(F_{x, n c}\right)}$

As we see, we only accelerate the critical transitions, the non-critical ones are handled in the same way as the repairs.

\subsection{Distance-based selected failure biasing (DSFB) [3]}

We assume that there may be some propagation of failures in the system. For all $x \in U$, its distance $d(x)$ to $D$ is defined as the minimal number of components whose failure put the model in a down state, that is,

$$
d(x)=\min _{y \in D} \sum_{k}\left(\nu_{k}(x)-\nu_{k}(y)\right) .
$$

Obviously, for any $y \in F_{x}$ we have $d(y) \leq d(x)$. A failure $(x, y)$ is said dominant if and only if $d(x)>d(y)$ and it is non-dominant iff $d(x)=d(y)$. The criticality of $(x, y) \in \mathcal{F}$ is

$$
c(x, y)=d(x)-d(y) \geq 0 .
$$

The idea of this algorithm is to take into account the different criticalities to control more deeply the failure transitions in the importance sampling scheme. It is assumed, of course, that the user can compute the distances $d(x)$ for any operational state $x$ with low cost.

Define recursively the following partition of $F_{x}$ :

$$
F_{x, 0}=\left\{y \in F_{x} \mid c(x, y)=0\right\}
$$

RR n 3672 
and $F_{x, l}$ is the set of states $y \in F_{x}$ such that $c(x, y)$ is the smallest criticality value greater than $c(x, w)$ for any $w \in F_{x, l-1}$. In symbols, if we denote, for all $l \geq 1$

$$
G_{x, l}=F_{x}-F_{x, 0}-F_{x, 1}-\cdots-F_{x, l-1}
$$

then we have

$$
F_{x, l}=\left\{y \in G_{x, l} \mid y=\operatorname{argmin}\left\{c(x, z), z \in G_{x, l}\right\}\right\}
$$

Let us denote by $V_{x}$ the number of criticality values greater than 0 of failures from $x$, that is,

$$
V_{x}=\operatorname{argmax}\left\{l \geq 1 \mid F_{x, l} \neq \emptyset\right\} .
$$

The method proposed in [3] has three parameters $\alpha, \beta, \beta_{c} \in(0,1)$. The new probability transitions are

- $\forall x \in U, \forall y \in F_{x, 0}, \quad f_{x}^{\prime}(y)=\alpha(1-\beta) \frac{f_{x}(y)}{f_{x}\left(F_{x, 0}\right)}$

$\forall l$ s.t. $1 \leq l<V_{x}, \forall y \in F_{x, l}, \quad f_{x}^{\prime}(y)=\alpha \beta\left(1-\beta_{c}\right) \beta_{c}^{l-1} \frac{f_{x}(y)}{f_{x}\left(F_{x, l}\right)}$

$\forall y \in F_{x, V_{x}}, \quad f_{x}^{\prime}(y)=\alpha \beta \beta_{c}^{V_{x}-1} \frac{f_{x}(y)}{f_{x}\left(F_{x, V_{x}}\right)}$

- $\forall x \neq 0, \forall y \in R_{x}, \quad r_{x}^{\prime}(y)=(1-\alpha) \frac{r_{x}(y)}{r_{x}\left(R_{x}\right)}$

As before, we must define what happens at the "frontiers" of the transformation. If $F_{x, 0}=\emptyset$, then we use $\beta=1$. If $x=0$, then we set $\alpha=1$.

It seems intuitively clear that we must, in general, give a higher weight to the failures with higher criticalities. This is not the case of the approach originally proposed in [3].

Just by "inverting" the order of the weights of the failures arriving at the $F_{x, l}, l \geq 1$, we obtain a new version which gives higher probabilities to failure transitions with higher criticalities. The Distance-based Selective Failure Biaising (DSFB) which we define here corresponds to the following algorithm: 
- $\forall x \in U, \forall y \in F_{x, 0}, \quad f_{x}^{\prime}(y)=\alpha(1-\beta) \frac{f_{x}(y)}{f_{x}\left(F_{x, 0}\right)}$

$\forall y \in F_{x, 1}, \quad f_{x}^{\prime}(y)=\alpha \beta \beta_{c}^{V_{x}-1} \frac{f_{x}(y)}{f_{x}\left(F_{x, 1}\right)}$

$\forall l$ s.t. $2 \leq l \leq V_{x}, \forall y \in F_{x, l}, \quad f_{x}^{\prime}(y)=\alpha \beta\left(1-\beta_{c}\right) \beta_{c}^{V_{x}-l} \frac{f_{x}(y)}{f_{x}\left(F_{x, l}\right)}$

- $\forall x \neq 0, \forall y \in R_{x}, \quad r_{x}^{\prime}(y)=(1-\alpha) \frac{r_{x}(y)}{r_{x}\left(R_{x}\right)}$

\subsection{Balanced methods}

All the previous methods classify the transitions from a fixed state into a number of disjoint sets, and assign modified global probabilities to each of these sets; but they do not modify the relative weights of the transitions belonging to the same set. An alternative is to assign uniform probabilities to all transitions from $x$ leading to the same subset of $F_{x}$. This can be done independently of the number and the definition of those sets, so that we can find balanced versions of all the previously mentioned methods.

Before looking the balanced versions in detail, let us observe that sometimes the systems are already "balanced" themselves, that is, there are no significant differences between the magnitude of the transition probabilities. In these cases, the unbalanced and balanced versions of the same method will basically behave in the same manner.

\section{Balanced FB}

Analyzing the FB method, it was proved (first in [14]) that balancing it improves its behaviour when there are transition probabilities from the same state $x$ which differ by orders of magnitude. The Balanced FB method is then defined by

- $\forall x \neq 0, \forall y \in F_{x}, \quad f_{x}^{\prime}(y)=\alpha \frac{1}{\left|F_{x}\right|}$

- $\forall x \neq 0, \forall y \in R_{x}, \quad r_{x}^{\prime}(y)=(1-\alpha) \frac{r_{x}(y)}{r_{x}\left(R_{x}\right)}$

RR n3672 
If $x=0$, then we set $\alpha=1$ in the algorithm.

\section{Balanced SFB}

The Balanced SFB scheme consists of the following rules:

- $\forall x \neq 0, \forall y \in I F_{x}, \quad f_{x}^{\prime}(y)=\alpha(1-\beta) \frac{1}{\left|I F_{x}\right|}$, and $\forall y \in N I F_{x}, \quad f_{x}^{\prime}(y)=\alpha \beta \frac{1}{\left|N I F_{x}\right|}$;

for $x=0$, we use the same formulae with $\alpha=1$; in the same way, if $I F_{x}=\emptyset$, we use $\beta=1$ and if $N I F_{x}=\emptyset$, we set $\beta=0$.

- $\forall x \neq 0, \forall y \in R_{x}, \quad r_{x}^{\prime}(y)=(1-\alpha) \frac{r_{x}(y)}{r_{x}\left(R_{x}\right)}$.

\section{Balanced SFBS}

We describe now the transformations associated with the Balanced SFBS scheme, except for the repairs and the frontier cases, which are as in the Balanced SFB's method:

- $\forall x, \forall y \in F_{x, n c}, \quad f_{x}^{\prime}(y)=\alpha(1-\beta) \frac{1}{\left|F_{x, n c}\right|}$, and $\forall y \in F_{x, c}, \quad f_{x}^{\prime}(y)=\alpha \beta \frac{1}{\left|F_{x, c}\right|}$.

\section{Balanced SFBP}

The Balanced SFBP method is defined by the following rules:

- $\forall x \in U, \forall y \in F_{x, c}, \quad f_{x}^{\prime}(y)=\alpha \frac{1}{\left|F_{x, c}\right|}$ and $\forall y \in F_{x, n c}, \quad f_{x}^{\prime}(y)=(1-\alpha) \frac{1}{\left|R_{x}\right|+\left|F_{x, n c}\right|}$.

- $\forall y \in R_{x}, \quad r_{x}^{\prime}(y)=(1-\alpha) \frac{1}{\left|R_{x}\right|+\left|F_{x, n c}\right|}$ 
It can be observed that, for the Balanced SFBP scheme, we do not take the repair probabilities proportionally to the original ones. Indeed, we have grouped repairs and non-initial failures, so taking the new transition probabilities proportional to the original ones would give rare events for the non-initial failures. Thus this small change, i.e. a uniform distribution over $F_{x, n c} \cup R_{x}$, balances all the transitions.

\section{Balanced DSFB}

The Balanced DSFB scheme is

- $\forall x \in U, \forall y \in F_{x, 0}, \quad f_{x}^{\prime}(y)=\alpha(1-\beta) \frac{1}{\left|F_{x, 0}\right|}\left(\right.$ as for $\left.B_{3}\right)$; $\forall y \in F_{x, 1}, \quad f_{x}^{\prime}(y)=\alpha \beta \beta_{c}^{V_{x}-1} \frac{1}{\left|F_{x, 1}\right|} ;$

$\forall l$ s.t. $2 \leq l \leq V_{x}, \forall y \in F_{x, l}, \quad f_{x}^{\prime}(y)=\alpha \beta\left(1-\beta_{c}\right) \beta_{c}^{V_{x}-l} \frac{1}{\left|F_{x, l}\right|}$;

- $\forall x \neq 0, \forall y \in R_{x}, \quad r_{x}^{\prime}(y)=(1-\alpha) \frac{r_{x}(y)}{r_{x}\left(R_{x}\right)}$

\section{Bounded relative error and bounded normal approximation}

In [14], Shahabuddin defines the concept of bounded relative error as follows:

Definition 6.1 Let $\sigma^{2}$ denote the variance of the estimator of $\gamma$ and $z_{\delta}$ the $1-\delta / 2$ quantile of the standard normal distribution. Then the relative error for a sample size $M$ is defined by

$$
R E=z_{\delta} \frac{\sqrt{\sigma^{2} / M}}{\gamma} .
$$

We say that we have a bounded relative error (BRE) if $R E$ remains bounded as $\varepsilon \rightarrow 0$.

RR n 3672 
If the estimator enjoys this property, only a fixed number of iterations is required to obtain a confidence interval having a fixed error no matter how rarely failures occur.

In $[16,15]$ the concept of bounded normal approximation is introduced to justify the use of the central limit theorem. Recall first the following version of the Berry-Esseen Theorem [1].

For a random variable $Z$, let $\varrho=E\left(|Z-E(Z)|^{3}\right), \sigma^{2}=E\left((Z-E(Z))^{2}\right)$ and let $\mathcal{N}$ be the standard normal distribution. For $Z_{1}, \cdots, Z_{I}$ i.i.d. copies of $Z$, define $\bar{Z}_{I}=I^{-1} \sum_{i=1}^{I} Z_{i}, \hat{\sigma}_{I}^{2}=I^{-1} \sum_{i=1}^{I}\left(Z_{i}-\bar{Z}_{I}\right)^{2}$ and let $F_{I}$ be the distribution of the centered and normalized sum $\left(Z_{1}+\cdots+Z_{I}\right) /\left(\hat{\sigma}_{I} \sqrt{I}\right)-E(Z) \sqrt{I} / \hat{\sigma}_{I}$. Then there exists an absolute constant $a>0$ such that, for each $x$ and $I$

$$
\left|F_{I}(x)-\mathcal{N}(x)\right| \leq \frac{a \varrho}{\sigma^{3} \sqrt{I}}
$$

Thus it is interesting to control the quantity $\varrho / \sigma^{3}$ because, in this way, the validity of the normal approximation, and then, of the coverage of the confidence interval, is guaranteed. A discussion on this point can be found in $[16,15]$. Following [16], we define the bounded normal approximation as follows.

Definition 6.2 If $\varrho$ denotes the third order moment and $\sigma$ the standard deviation of the estimator of $\gamma$, we say that we have a bounded normal approximation (BNA) if $\varrho / \sigma^{3}$ is bounded when $\varepsilon \rightarrow 0$.

Necessary and sufficient conditions for both properties are known ([12] for BRE and [15, 16] for BNA). It has been proven (see [12], [14]) that Balanced FB leads to the BRE property and it has been also shown that this is not true for unbalanced methods. Similarly, it can be shown ([14], or using [12, Theorem 2]) that any of the balanced algorithms gives BRE. Moreover, the following result holds.

Theorem 6.3 [15, 16] If we have BNA, we have BRE. Nevertheless, there exist systems with BRE but without BNA.

This means that we must not only check for the BRE property: the critical one is BNA. It is also proven in [16] and in [15] that any balanced method 
verifies the BNA property, so balancing all the methods leads to good properties. Using the necessary and sufficient conditions for BRE and BNA, i.e. [12, Theorem 2] and [16, Theorem 4], it is immediate to see that, in fact, any change of measure independent of the rarity parameter $\varepsilon$ verifies the BRE and BNA properties (for the BRE property, this has been observed first in [14]).

\section{Asymptotic comparison of methods}

Given a specified system, we can wonder which scheme, among the several ones described in Section 5, is the most appropriate. This section has two folds. First, we explain why we do not use a $\beta$ parameter in the SFBP scheme, as we do in the SFB and SFBS cases. Second, we make some asymptotic comparisons of the discussed techniques. We consider only balanced schemes because they are the only ones, among the methods described in Section 5, to verify in general the BRE and BNA properties.

The asymptotic efficiency (as $\varepsilon \rightarrow 0$ ) is controlled by two quantities: the asymptotic variance of the estimator and the mean number of transitions needed by embedded chain $Y$ to hit $D$ when it does it before coming back to 0 .

\subsection{On the SFBP choice}

- We want to compare the variance of the two considered choices in SFBP (with or without a $\beta$ parameter), in the case of a system structured as a set of $l_{k}$-out-of- $N_{k}$ modules in parallel, $k=1, \cdots, K$, i.e. the case of interest. To do this, let us denote by $f_{x, \beta}^{\prime}(y)$ the transition probability associated with a SFBP scheme using a $\beta$ parameter, as shown before, in the first part of 5.4. Let $s$ be the integer such that $\sigma^{2}=\Theta\left(\varepsilon^{s}\right)$. We can observe that the most important paths for the variance estimation, i.e. the paths $c \in \mathcal{D}$ verifying $q^{2}(c) / q^{\prime}(c)=\Theta\left(\varepsilon^{s}\right)$ are typically composed of critical transitions $(x, y)$ for which the failure SFBP probability $f_{x}^{\prime}(y)$ (without using $\beta$ ) verify

$$
f_{x}^{\prime}(y)=f_{x, \beta}^{\prime}(y) / \beta
$$

RR n3672 
i.e., transitions driving closer to the failure states. So, if we note $\sigma^{2}$ (resp.

$\left.\sigma_{\beta}^{2}\right)$ the variance of the estimator without (resp. with) the $\beta$ parameter, $\sigma^{2} / \sigma_{\beta}^{2}<1$ as $\varepsilon \rightarrow 0$.

- Let us denote by $|c|$ the number of transitions in cycle $c \in \mathcal{D}$ until hitting $D$. The expected number of transitions necessary to hit $D$ under the modified measure $q^{\prime}$ is

$$
E(T)=\sum_{c \in \mathcal{D}}|c| q^{\prime}(c)
$$

From relation (2), we see that $E(T)$ is smaller if we do not use the $\beta$ parameter.

From both of these points of view, we conclude that not using a $\beta$ parameter in SFBP scheme is a good idea.

\subsection{Comparison of Balanced schemes}

Using the balanced schemes, all the variances are of the same order (i.e. $O\left(\varepsilon^{2 r}\right)$ ) because each path is in $\Theta(1)$ (see [14] or [12] for a proof). Then, we can point out the following facts:

- The variances are of the same order with all the balanced schemes. Nevertheless the constant of the $\Theta(\cdot)$ may be quite different. The analysis of this constant is much more difficult in this general case than for the SFBP schemes previously presented and appears to depend too much on the specific model parameters to allow any kind of general claim about it.

- The preceding point suggests basing the choice between the different methods mainly on the mean hitting time to $D$ given in (3). To get the shortest computational time, our heuristic is the following:

- if there are many propagation faults in the system, we suggest the use of a Balanced DSFBP scheme; 
- if there is no (or very few) propagation faults and if the system is working as a series of $l_{k}$-out-of- $N_{k}$ modules, the balanced SFBS scheme seems the appropriate one;

- if there no (or very few) propagation faults and if the system is working as a set of $l_{k}$-out-of- $N_{k}$ modules in parallel, $1 \leq k \leq K$, we suggest the use of the Balanced SFBP method;

- it remains the case of a poorly structured system, or one where it is not clear if the structure function is rather of the series type, or of the parallel one; in those cases, the general Balanced FB scheme can also lead to a useful variance reduction.

\section{Numerical illustrations}

All the systems used in the numerical illustrations given in this section were modeled and evaluated using a specific library (called BB as balls 8 buckets framework) [2], on a SPARCstation 10 Model 602 workstation. In all cases, the estimated measure is $\gamma=\operatorname{Pr}\left(\tau_{D}<\tau_{0}\right)$.

We are not going to compare all the methods discussed before in both versions, unbalanced and balanced. Our aim is to get a feeling of what can be obtained in practice, and to give some general guidelines to choose among the different methods.

First, let us consider methods FB, SFB and SFBS. When the modeled systems have a structure close to a series of $l_{k}$-out-of- $N_{k}$ modules, it seems clear that both SFB and SFBS are better than FB. If the values $N_{k}-l_{k}$ (that is, the number of redundant components for class $k$ ) do not (or do slightly) depend on $k$, SFB and SFBS should have more or less the same behaviour; but when some components have significant differences in these values, SFBS should outperform SFB. To look at how these rules of thumb work out in a particular case, we study two versions of a Tandem computer, described in [7] (we follow here a later description in [9]). This computer is composed of a multiprocessor $p$, a dual disk controller $k$, two RAID disk drives $d$, two fans $f$, two power supplies $p s$, and one dual interprocessor bus $b$. In addition to a CPU, each processor contains its own memory. When a component in a dual fails, the subsystem is reconfigured into a simplex. This Tandem computer requires

RR n 3672 
all subsystems, one fan, and one power supply for it to be operational. The failure rates $\lambda_{k}(x)$ are $5 \varepsilon, 2 \varepsilon, 4 \varepsilon, 0.1 \varepsilon, 3 \varepsilon$ and $0.3 \varepsilon$ for the processors, the disk controller, the disks, the fans, the power supplies and the bus respectively, with $\varepsilon=10^{-5} \mathrm{f} / \mathrm{hr}$. There is only one repairman and the repair rates are $\mu_{k}(x)=30 \mathrm{r} / \mathrm{hr}$, for all the components, except for the bus, which has repair rate $\mu_{k}(x)=15 \mathrm{r} / \mathrm{hr}$.

We first consider a version of this computer where both the multiprocessor and the disks have two units, and only one is needed for the system to be working. In this case, $N_{k}=2$ and $l_{k}=1$ for all $k$. Table I presents the variances and computing times for the FB, the SFB and the SFBS methods, observed when estimating $\gamma$ with a sample size $M=10^{5}$, and parameters $\alpha=0.7, \beta=$ 0.8. As expected, we can observe that for this situation, algorithms SFB and SFBS are equivalent (both in precision and in execution time); their variance is an order of magnitude better than the variance of the $\mathrm{FB}$ algorithm, which is also slower. The slight difference in the execution time between SFB and SFBS comes from the fact that in the latter there is a little bit of supplementary computations to do, with basically the same cycle structure.

\begin{tabular}{|l|c|c|}
\hline Method & Variance & Time (sec.) \\
\hline FB & $2.98 \times 10^{-16}$ & 113 \\
SFB & $3.43 \times 10^{-17}$ & 60 \\
SFBS & $3.43 \times 10^{-17}$ & 64 \\
\hline
\end{tabular}

Table I: Methods FB, SFB, and SFBS for a series $h_{k}$-out-of- $N_{k}$ system with no dependence on $k$

Let us now consider this same architecture, but with with a four-unit multiprocessor (only one of the four processors is required to have an operational system); and with each RAID being composed by 5 drives, only 3 of which are required. In this case, $N_{k}$ and $l_{k}$ vary for different $k$. Table II presents the variances and computing times for the FB, the SFB and the SFBS methods, observed when estimating $\gamma$ with a sample size $M=10^{5}$, and parameters $\alpha=0.7, \beta=0.8$. As in the previous case, the FB algorithm is the least performant; but now we observe how SFBS obtains a better precision (at a lower computational cost) than SFB. 


\begin{tabular}{|l|c|l|}
\hline Method & Variance & Time (sec.) \\
\hline FB & $5.90 \times 10^{-18}$ & 318 \\
SFB & $9.23 \times 10^{-19}$ & 170 \\
SFBS & $6.20 \times 10^{-19}$ & 146 \\
\hline
\end{tabular}

Table II: Methods FB, SFB and SFBS for a series $h_{k}$-out-of- $N_{k}$ system with dependence on $k$

Consider now a model of a replicated database; there are four sites, and each site has a whole copy of the database, on a RAID disk cluster. We take all clusters identical, with the same redundancies (7-out-of-9), and with failure rate (for each disk) of $\varepsilon=10^{-2}$. There is one repairman per class, and the repair rate is 1 . We consider that the system is up if there is at least one copy of the database in working order: then the structure function of this system is a parallel $l_{k}$-out-of- $N_{k}$. We compare in Table III the behaviour of FB, SFB, and SFBP algorithms for this system, where all component classes $k$ have the same redundancy; the SFBP method performs much better than both FB and SFB.

\begin{tabular}{|l|c|l|}
\hline Method & Variance & Time (sec.) \\
\hline FB & $2.17 \times 10^{-27}$ & 398 \\
SFB & $8.74 \times 10^{-26}$ & 450 \\
SFBP & $8.89 \times 10^{-28}$ & 267 \\
\hline
\end{tabular}

Table III: Methods FB, SFB, and SFBP for a parallel $h_{k}$-out-of- $N_{k}$ system with no dependence on $k$

Consider now a model with failure propagation, the fault-tolerant database system presented in [11]. The components of this system are: a front-end, a database, and two processing subsystems formed by a switch, a memory, and two processors. These components may fail with rates $1 / 2400,1 / 2400,1 / 2400$, $1 / 2400$ and $1 / 120$ respectively. There is a single repairman who gives priority to the front-end and the database, followed by the switches and memory units, followed by the processors; all with repair rate 1 . If a processor fails it contaminates the database with probability .001. The systems is operational if the front-end, the database, and a processing subsystem are up; a processing sub-

RR n 3672 
system is up if the switch, the memory, and a processor are up. We illustrate in Table IV the results obtained with the FB, SFB, and DSFB techniques using $\alpha=0.7, \beta=0.8, \beta_{c}=0.2$, for a sample size $M=10^{5}$. The DSFB technique is much superior in this context, both in precision (a two-order reduction in the variance) and in computational effort. Its reduced execution time is due to the fact that, going much faster to the states where the system is down than with the other methods, the cycle lengths are much shorter.

\begin{tabular}{|l|c|c|}
\hline Method & Variance & Time (sec.) \\
\hline FB & $1.014 \times 10^{-6}$ & 116 \\
SFB & $1.016 \times 10^{-6}$ & 120 \\
DSFB & $2.761 \times 10^{-8}$ & 47 \\
\hline
\end{tabular}

Table IV: Methods FB, SFB, and DSFB for a system with failure propagations

Our last example illustrates the use of simulation techniques to evaluate a model with a very large state space. The system is similar to one presented in [13], but has more components and as a result the underlying Markov chain has a larger state space. The system is composed of two sets of 4 processors each, 4 sets of 2 dual-ported controllers, and 8 sets of disk arrays composed by 4 units. Each controller cluster is in charge of 2 disk arrays; each processor has access to all the controllers clusters. The system is up if there are at least one processor (of either class), one controller of each cluster, and three disks of each array, in operational order.

The failure rates for the processors and the controllers are 1/2000; for the disk arrays we consider four different failure rates (each corresponds to two arrays), namely $1 / 4000,1 / 5000,1 / 8000$ and $1 / 10000$. a single case of failure propagation: when a processor of a cluster fails, there is a 0.10 probability that a processor of the other cluster is affected. Each failure has two modes; the repair rates depend on the mode, and take the value 1 for the first mode and 0.5 for the second.

The system has more than $7.4 \times 10^{14}$ states in its state space; this precludes even the generation of the state space, and makes it impossible to think of using any exact techniques.

We illustrate in Table $\mathrm{V}$ the results obtained with the crude, FB, SFB and DSFB techniques using $\alpha=0.7, \beta=0.8, \beta_{c}=0.2$, for a sample size $M=10^{5}$. 
Since this is a complex case the execution times are larger than those observed for the previous cases; but even the slowest method, FB, takes less than 27 minutes to complete the experiment. In all cases, when using importance sampling techniques the variances obtained are between 2 and 3 orders of magnitude smaller than the variance of the crude simulation technique; this allows to estimate $\gamma$ to a higher precision with the same number of replications. The technique which a priori seems the more appropriate to this kind of system with failure propagations is DSFB; the experimental results confirm this, as DSFB not only has the best variance, but also the best execution time among the importance sampling techniques compared, and only twice the execution time of the crude technique.

The data numerical values in this example have been chosen such that with the relatively small number $M$ of iterations, even the crude method allows to obtain a confidence interval. At the same time, this allows to underline the importance of the concept of efficiency: even FB is more efficient than the crude technique, since we must take into account both the execution time and the obtained precision.

\begin{tabular}{|l|c|c|c|}
\hline Method & $95 \%$ confidence interval for $\gamma$ & Variance & Time (sec.) \\
\hline crude & {$\left[2.257 \times 10^{-4}, 4.542 \times 10^{-4}\right]$} & $3.399 \times 10^{-9}$ & 232 \\
FB & {$\left[2.448 \times 10^{-4}, 2.704 \times 10^{-4}\right]$} & $2.247 \times 10^{-11}$ & 1586 \\
SFB & {$\left[2.577 \times 10^{-4}, 2.641 \times 10^{-4}\right]$} & $2.607 \times 10^{-12}$ & 973 \\
DSFB & {$\left[2.601 \times 10^{-4}, 2.644 \times 10^{-4}\right]$} & $1.189 \times 10^{-12}$ & 461 \\
\hline
\end{tabular}

Table V: Crude, FB, SFB and DSFB methods for a very large system

\section{Some numerical aspects of rarity}

Let us consider a very simple system with 2 components, one of class 1 , one of class 2 . The state space is $S=\{0,1,2,3\}$ where 0 is the state with both components up, in state 1 the component of class 1 is down and the other one is up, 2 represents the opposite situation and in state 3 , both components are down. We do not make any particular assumption about the repairs. Assume that the probability transitions verify $P(0,1) \approx \varepsilon, P(0,2) \approx 1, P(1,3) \approx \varepsilon$ and $P(2,3) \approx \varepsilon^{2}$.

RR n 3672 
There are 4 cycles: $c_{1}=(0,1,3), c_{2}=(0,2,3), c_{3}=(0,1,0)$ and $c_{4}=$ $(0,2,0)$. The set of cycles through $D$ is $\mathcal{D}=\left\{c_{1}, c_{2}\right\}$. The respective probabilities are $q\left(c_{1}\right) \approx \varepsilon^{2}, q\left(c_{2}\right) \approx \varepsilon^{2}, q\left(c_{3}\right) \approx \varepsilon$ and $q\left(c_{4}\right) \approx 1$. Consider the FB basic technique. After the measure change, the new probability transitions are: $P^{\prime}(0,1) \approx \varepsilon, P^{\prime}(0,2) \approx 1, P^{\prime}(1,3)=\alpha$ and $P^{\prime}(2,3)=\alpha$, leading to the new cycle probabilities $q^{\prime}\left(c_{1}\right) \approx \alpha \varepsilon, q^{\prime}\left(c_{2}\right) \approx \alpha, q^{\prime}\left(c_{3}\right) \approx(1-\alpha) \varepsilon$ and $q^{\prime}\left(c_{4}\right) \approx 1-\alpha$.

Computing $\gamma$ gives $\gamma \approx 2 \varepsilon^{2}$ and $\sigma^{2} \approx \varepsilon^{3} / \alpha$. This implies that we do not have bounded relative error since

$$
\frac{\sigma}{\gamma} \approx \frac{1}{\sqrt{2 \alpha}} \varepsilon^{-\frac{1}{2}} .
$$

Since the analysis is done for a fixed number $M$ of trials, the equivalents of the $q^{\prime}\left(c_{i}\right)$ 's show that for $\varepsilon$ small enough, it will be very unlikely that cycles $c_{1}$ and $c_{3}$ are sampled under $q^{\prime}$. We will obtain approximatively $M \alpha$ samples of cycle $c_{2}$ and $M(1-\alpha)$ samples of cycle $c_{4}$. Denoting by $L_{m}$ the "weighted" likelihood of the $m^{\text {th }}$ replication, that is,

$$
L_{m}=\frac{q\left(C_{m}\right)}{q^{\prime}\left(C_{m}\right)} 1_{\left(\tau_{D}-\tau_{0}\right)}\left(C_{m}\right),
$$

the estimation of the relative error is

$$
\begin{aligned}
\widehat{R E} & =z_{\delta} \sqrt{\frac{M}{M-1} \sum_{m=1}^{M} \frac{L_{m}^{2}}{\left(\sum_{i=1}^{M} L_{i}\right)^{2}}-\frac{1}{M-1}} \\
& \approx z_{\delta} \sqrt{\frac{M}{M-1} \alpha M \frac{\left(\varepsilon^{2} / \alpha\right)^{2}}{\left(\alpha M \varepsilon^{2} / \alpha\right)^{2}}-\frac{1}{M-1}} \\
& =z_{\delta} \sqrt{\frac{1}{(M-1) \alpha}-\frac{1}{M-1} .}
\end{aligned}
$$

In other words, the observed relative error $\widehat{R E}$ is bounded while the theoretical $R E$ is not. This is, again, a negative consequence of the rare event situation. This is also a supplementary reason to only use balanced importance sampling methods. 


\section{Conclusion}

We discussed the best known methods designed to estimate the MTTF of a complex system modeled by a Markov chain, in the rare events context. We propose new versions of some of these algorithms, behaving better than the original ones in some situations. We also analyzed the main properties of the considered techniques: the bounded relative error concept, the bounded normal approximation concept, their relationships and the relationships with the balanced versions of the estimation algorithms. The discussion should be helpful in (i) choosing among the available techniques and (ii) in designing new variance reduction algorithms for the same or for other dependability measures.

\section{References}

[1] V. Bentkus and F. Götze. The Berry-Esseen bound for Student's statistic. The Annals of Probability, 24(1):491-503, 1996.

[2] H. Cancela. Évaluation de la sûreté de fonctionnement: modèles combinatoires et Markoviens. PhD thesis, Université de Rennes 1, December 1996.

[3] J. A. Carrasco. Failure distance based on simulation of repairable fault tolerant systems. In Proceedings of the 5th International Conference on Modelling Techniques and Tools for Computer Performance Evaluation, pages 351-365, 1991.

[4] A.E. Conway and A. Goyal. Monte Carlo simulation of computer system availability/reliability models. In Proceedings of the Seventeenth Symposium on Fault-Tolerant Computing, pages 230-235, July 1987.

[5] A. Goyal, P. Shahabuddin, P. Heidelberger, V. F. Nicola, and P. W. Glynn. A unified framework for simulating Markovian models of highly dependable systems. IEEE Transactions on Computers, 41(1):36-51, January 1992.

RR n 3672 
[6] P. Heidelberger. Fast simulation of rare events in queueing and reliability models. ACM Transactions on Modeling and Computer Simulations, 54(1):43-85, January 1995.

[7] J. Katzmann. System architecture for non-stop computing. In 14th IEEE Comp. Soc. International Conf, pages 77-80, 1977.

[8] E.E. Lewis and F. Böhm. Monte Carlo simulation of Markov unreliability models. Nuclear Engineering and Design, 77:49-62, 1984.

[9] C. Liceaga and D. Siewiorek. Automatic specification of reliability models for fault tolerant computers. NASA technical paper 3301, July 1993.

[10] S. Mahévas and G. Rubino. Bound computation of dependability and performance measures. IEEE Transactions on Computers, to appear in 1999.

[11] R.R. Muntz, E. de Souza e Silva, and A. Goyal. Bounding availability of repairable computer systems. IEEE Transactions on Computers, 38(12):1714-1723, 1989.

[12] M. K. Nakayama. General Conditions for Bounded Relative Error in Simulations of Highly Reliable Markovian Systems. Advances in Applied Probability, 28:687-727, 1996.

[13] V. F. Nicola, M. K. Nakayama, P. Heidelberger, and A. Goyal. Fast simulation of highly dependable systems with general failure and repair processes. IEEE Transactions on Computers, 42(12):1440-1452, December 1993 .

[14] P. Shahabuddin. Importance sampling for the simulation of highly reliable Markovian systems. Management Science, 40(3):333-352, March 1994.

[15] B. Tuffin. Simulation accélérée par les méthodes de Monte Carlo et quasiMonte Carlo: théorie et applications. $\mathrm{PhD}$ thesis, Université de Rennes 1, October 1997. 
[16] B. Tuffin. Bounded Normal Approximation in Highly Reliable Markovian Systems. Technical Report 1191 (updated version of INRIA Research Report 3020 of October 1996), IRISA, May 1998. ftp://ftp.irisa.fr/pub/techreports/1998/PI-1191.ps.gz, to appear in JAP, 1999.

RR n3672 


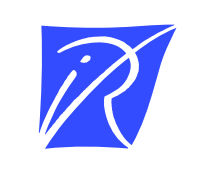

Unit'e de recherche INRIA Lorraine, Technopôle de Nancy-Brabois, Campus scientifique, 615 rue du Jardin Botanique, BP 101, 54600 VILLERS LÈS NANCY

Unit'e de recherche INRIA Rennes, Irisa, Campus universitaire de Beaulieu, 35042 RENNES Cedex

Unit'e de recherche INRIA Rhône-Alpes, 655, avenue de l'Europe, 38330 MONTBONNOT ST MARTIN

Unit'e de recherche INRIA Rocquencourt, Domaine de Voluceau, Rocquencourt, BP 105, 78153 LE CHESNAY Cedex

Unit'e de recherche INRIA Sophia-Antipolis, 2004 route des Lucioles, BP 93, 06902 SOPHIA-ANTIPOLIS Cedex

Éditeur

INRIA, Domaine de Voluceau, Rocquencourt, BP 105, 78153 LE CHESNAY Cedex (France)

http://www.inria.fr

ISSN 0249-6399 